\title{
HERSCHEL OBSERVATIONS REVEAL ANOMALOUS MOLECULAR ABUNDANCES TOWARD THE GALACTIC CENTER
}

\author{
P. Sonnentrucker ${ }^{1}$, D. A. Neufeld ${ }^{2}$, M. Gerin ${ }^{3}$, M. De LucA $^{3}$, N. Indriolo ${ }^{2}$, D. C. Lis ${ }^{4}$, And J. R. GoicoecheA \\ ${ }^{1}$ Space Telescope Science Institute, Baltimore, MD 21218, USA; sonnentr@ stsci.edu \\ ${ }^{2}$ Physics and Astronomy Department, Johns Hopkins University, Baltimore, MD 21218, USA \\ ${ }^{3}$ LERMA-LRA, UMR 8112 du CNRS, Observatoire de Paris, École Normale Supérieure, UPMC \& UCP, 24 rue Lhomond, F-75231, Paris Cedex 05, France \\ ${ }^{4}$ Astronomy Department, California Institute of Technology, Pasadena, CA 91125, USA \\ ${ }_{5}^{5}$ Centro de Astrobiología, CSIC/INTA, E-28850, Madrid, Spain \\ Received 2012 September 4; accepted 2012 December 11; published 2013 January 7
}

\begin{abstract}
We report the Herschel detections of hydrogen fluoride $(\mathrm{HF})$ and para-water $\left(\mathrm{p}-\mathrm{H}_{2} \mathrm{O}\right)$ in gas intercepting the sight lines to two well-studied molecular clouds in the vicinity of the Sgr A complex: G-0.02-0.07 (the

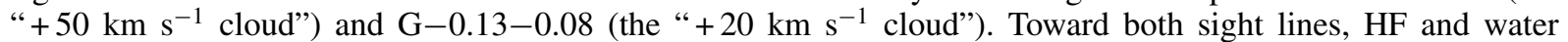
absorption components are detected over a wide range of velocities covering $\sim 250 \mathrm{~km} \mathrm{~s}^{-1}$. For all velocity components with $V_{\mathrm{LSR}}>-85 \mathrm{~km} \mathrm{~s}^{-1}$, we find that the $\mathrm{HF}$ and water abundances are consistent with those measured toward other sight lines probing the Galactic disk gas. The velocity components with $V_{\mathrm{LSR}} \leqslant-85 \mathrm{~km} \mathrm{~s}{ }^{-1}$, which are known to trace gas residing within $\sim 200 \mathrm{pc}$ of the Galactic center, however, exhibit water vapor abundances with respect to $\mathrm{HF}$ at least a factor three higher than those found in the Galactic disk gas. Comparison with $\mathrm{CH}$ data indicates that our observations are consistent with a picture where $\mathrm{HF}$ and a fraction of the $\mathrm{H}_{2} \mathrm{O}$ absorption arise in diffuse molecular clouds showing Galactic disk-like abundances while the bulk of the water absorption arises in warmer $(T \geqslant 400 \mathrm{~K})$ diffuse molecular gas for $V_{\mathrm{LSR}} \leqslant-85 \mathrm{~km} \mathrm{~s}^{-1}$. This diffuse Interstellar Medium (ISM) phase has also been recently revealed through observations of $\mathrm{CO}, \mathrm{HF}, \mathrm{H}_{3}^{+}$, and $\mathrm{H}_{3} \mathrm{O}^{+}$absorption toward other sight lines probing the Galactic center inner region.
\end{abstract}

Key words: ISM: individual objects (G, 0.02, 0.07, G, 0.13, 0.08) - ISM: lines and bands - ISM: molecules

\section{INTRODUCTION}

The detection of atomic (e.g., Genzel et al. 1990; Esteban et al. 2005; Lang et al. 2010) and molecular species within $\sim 200 \mathrm{pc}$ around the Galactic center has revealed the coexistence of cold dense molecular clouds (e.g., Guesten et al. 1981), warm dense molecular clouds (e.g., Rodriguez-Fernandez et al. 2006), or cold diffuse clouds (e.g., Lang et al. 2010). Their distribution within the three major structures known to exist in this inner region - the circumnuclear disk (CND), the central molecular zone (CMZ), and the extended molecular ring (EMR) - remains subject to extensive studies and significant debate to date (e.g., Wright et al. 2001; Dwarakanath et al. 2004). $\mathrm{H}_{3}{ }^{+}$detections toward discrete sight lines probing the CMZ (e.g., Oka et al. 2005,2011 ) also revealed the presence of a warm diffuse phase unique to the Galactic center's inner region. Our $\mathrm{H}_{2} \mathrm{O}$ and $\mathrm{HF}$ observations combined with the results of Monje et al. (2011) toward Sgr B2 establish that this newly detected warm and very dilute phase seems pervasive around the Galactic center.

As part of the Guaranteed Time Key Program PRISMAS ("PRobing the Interstellar Medium with Absorption line Studies"; PI: M. Gerin), we observed two well-studied molecular clouds in the Sgr A complex: G-0.02-0.07 (the " $+50 \mathrm{~km} \mathrm{~s}^{-1}$ cloud") and G-0.13-0.08 (the " $+20 \mathrm{~km} \mathrm{~s}^{-1}$ cloud") with the Heterodyne Instrument for the Far-Infrared (HIFI) on Herschel (see Pilbratt et al. 2010). Toward both sight lines we see strong absorption from hydrogen fluoride (HF) and para-water $\left(\mathrm{p}-\mathrm{H}_{2} \mathrm{O}\right)$, consisting of a series of gas components residing in the foreground of the Galactic center or gas components originating in the inner $200 \mathrm{pc}$ region of Sgr A. This Letter focuses on the unusual conditions in the gas components arising within $200 \mathrm{pc}$ of the Galactic center (e.g., Lang et al. 2010; Dwarakanath et al. 2004). The gas components arising in this foreground Galactic disk material will be discussed in a subsequent paper presenting $\mathrm{HF}$ and water detections toward the remaining PRISMAS targets W28, G34.1, and DR21(OH) (P. Sonnentrucker et al. 2013, in preparation). Section 2 describes the Herschel observations along with our data reduction procedures. Section 3 discusses our $\mathrm{HF}$ and $\mathrm{p}-\mathrm{H}_{2} \mathrm{O}$ results. Finally, Section 4 offers an interpretation for the increased water vapor abundance within the $\mathrm{CMZ}$ relative to the Galactic disk using $\mathrm{CH}$ observations obtained with Herschel toward our sight lines.

\section{OBSERVATIONS AND DATA ANALYSIS}

We observed the ground-state rotational line of $\mathrm{HF}\left(v_{\text {rest }}=\right.$ $1232.476 \mathrm{GHz}$ ) in the upper sideband of HIFI band 5 a receiver and the ground-state line of $\mathrm{p}-\mathrm{H}_{2} \mathrm{O}\left(v_{\text {rest }}=1113.343 \mathrm{GHz}\right)$ in the lower sideband of HIFI band 5a receiver using multiple Local Oscillator (LO) settings in order to securely identify the $\mathrm{HF}$ and $\mathrm{p}-\mathrm{H}_{2} \mathrm{O}$ absorption lines toward $\mathrm{G}-0.02-0.07$ and $\mathrm{G}-0.13-0.08$. The proximity of the $\mathrm{HF}$ and $\mathrm{p}-\mathrm{H}_{2} \mathrm{O}$ lines in frequency and their detection within the same receiver band ensure that the absorption lines are observed with a very similar beam size and that the data are calibrated in a similar fashion. We used the Dual Beam Switch (DBS) mode which, combined with the Wide Band Spectrometer (WBS), allows for a spectral resolution of about $1.1 \mathrm{MHz}\left(0.3 \mathrm{~km} \mathrm{~s}^{-1}\right.$ at the $\mathrm{HF}$ frequency). The DBS mode uses a reference OFF-beam position located $3^{\prime}$ on either side of the source position along an east-west axis. Because the Galactic center is a very complex and crowded region, we checked for contamination in the OFF-beam position using Herschel observations performed by the HEXGAL project (R. Güsten \& M. Requena-Torres 2012, private communication). There is no contamination at the OFF-beam position for HF; only minor contamination occurs for $\mathrm{p}-\mathrm{H}_{2} \mathrm{O}$ in the velocity range $[-40,+10] \mathrm{km} \mathrm{s}^{-1}$. 
Table 1

Summary of Observations

\begin{tabular}{|c|c|c|c|c|c|c|c|}
\hline Target & $\begin{array}{c}\text { R.A. }^{\mathrm{a}} \\
(\mathrm{J} 2000.1)\end{array}$ & $\begin{array}{c}\text { Decl. }^{\mathrm{a}} \\
(\mathrm{J} 2000.1)\end{array}$ & $\begin{array}{c}T_{\exp }(\mathrm{HF}) \\
\text { (s) }\end{array}$ & $\begin{array}{c}T_{\mathrm{A}}(\mathrm{cont})[\mathrm{HF}] \\
(\mathrm{K})\end{array}$ & $\begin{array}{c}T_{\exp }\left(\mathrm{H}_{2} \mathrm{O}\right) \\
\text { (s) }\end{array}$ & $\begin{array}{c}T_{\mathrm{A}}(\text { cont })\left[\mathrm{H}_{2} \mathrm{O}\right] \\
(\mathrm{K})\end{array}$ & Obs. Date ${ }^{b}$ \\
\hline $\mathrm{G}-0.02-0.07$ & $17^{\mathrm{h}} 45^{\mathrm{m}} 50^{\mathrm{s}} .200$ & $-28^{\mathrm{d}} 59^{\mathrm{m}} 53^{\mathrm{s}} .00$ & 3693 & $0.92 \pm 0.05$ & 2139 & $0.85 \pm 0.05$ & 2010 Oct 6 \\
\hline$G-0.13-0.08$ & $17^{\mathrm{h}} 45^{\mathrm{m}} 37^{\mathrm{s}} .400$ & $-29^{\mathrm{d}} 05^{\mathrm{m}} 40^{\mathrm{s}} .00$ & 746 & $1.38 \pm 0.10$ & 428 & $1.47 \pm 0.10$ & 2011 Sep 14 \\
\hline
\end{tabular}

Notes.

a Coordinates of the center of the telescope beam.

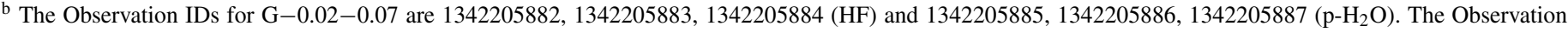
IDs for G-0.13-0.08 are: $1342228613,1342228614(\mathrm{HF})$ and $1342228615,1342228616\left(\mathrm{p}-\mathrm{H}_{2} \mathrm{O}\right)$.

We processed the data to Level 2 with the standard HIFI pipeline in the Herschel Interactive Processing Environment (HIPE) version 7.3.0 (Ott 2010), thereby producing fully calibrated spectra for both polarization modes at each LO setting. We analyzed the Level 2 data in a fashion identical to that described in Sonnentrucker et al. (2010). Table 1 lists, for both sight lines, the target name, the coordinates of the center of the telescope beam, the total on-source exposure times, the double sideband continuum antenna temperatures, the observation dates, and the observation IDs for the multiple LO settings we obtained for $\mathrm{HF}$ and $\mathrm{p}-\mathrm{H}_{2} \mathrm{O}$.

HIFI employs double sideband receivers. Thus, for a sideband gain ratio equal to unity, the saturated absorption of radiation at a given frequency will reduce the measured antenna temperature $\left(T_{\mathrm{A}}\right)$ to one-half the apparent continuum antenna temperature $T_{\mathrm{A}}$ (cont). Our observations, however, indicate that the reduction in the measured antenna temperature for complete absorption of radiation at given frequencies is greater than one-half the continuum temperature by $5 \%$ for $\mathrm{G}-0.02-0.07$ and by about $8 \%$ for $\mathrm{G}-0.13-0.08$. We took these correction factors - which arise from a sideband gain ratio slightly different from unity-into account when normalizing the flux with respect to the continuum flux in a single sideband.

\section{RESULTS}

The left panels of Figure 1 display the normalized singlesideband line intensities versus $V_{\mathrm{LSR}}$ for HF (black solid line) and $\mathrm{p}-\mathrm{H}_{2} \mathrm{O}$ (green solid line) toward $\mathrm{G}-0.02-0.07$ (top) and $\mathrm{G}-0.13-0.08$ (bottom). The right panels of Figure 1 compare the optical depth profiles of $\mathrm{HF}$ (black) and $\mathrm{p}-\mathrm{H}_{2} \mathrm{O}$ (green) derived from our spectra in the LSR velocity range we focus on in this study $\left([-200,-40] \mathrm{km} \mathrm{s}^{-1}\right)$ for $\mathrm{G}-0.02-0.07$ (top) and $\mathrm{G}-0.13-0.08$ (bottom). The velocity distribution of both molecules follows the general gas kinematics derived from previous line studies toward the Galactic center. In particular, the components with $-60 \leqslant V_{\mathrm{LSR}} \leqslant+10 \mathrm{~km} \mathrm{~s}^{-1}$ arise predominantly in foreground clouds located in the $3 \mathrm{kpc}$ Galactic Arm (e.g., Lang et al. 2010; Rodriguez-Fernandez et al. 2006). The gas components with $-130 \leqslant V_{\mathrm{LSR}} \leqslant-70 \mathrm{~km} \mathrm{~s}^{-1}$ arise in a CND with a projected radius of $\sim 17$ pc surrounding Sgr A (e.g., Liszt et al. 1985; Oka et al. 2011) while the components with $-200 \leqslant V_{\mathrm{LSR}} \leqslant-70 \mathrm{~km} \mathrm{~s}^{-1}$ arise predominantly in the CMZ, a molecular region within 200 pc of Sgr A also engulfing the CND (e.g., Liszt et al. 1983; Sawada et al. 2004).

We see striking similarity in the velocity and optical depth distributions of $\mathrm{HF}$ and $\mathrm{p}-\mathrm{H}_{2} \mathrm{O}$ for the components with $-60 \mathrm{~km} \mathrm{~s}^{-1} \leqslant V_{\mathrm{LSR}} \leqslant-40 \mathrm{~km} \mathrm{~s}^{-1}$. Such similarities are observed now routinely toward other Galactic sight lines (W31C, Neufeld et al. 2010; W49N and W51, Sonnentrucker et al. 2010) and indicate that these particular gas components have abun- dances consistent with those in the Galactic disk whether they are mainly tracing gas in the foreground of the Galactic center or whether they also trace gas local to the Galactic center. While the velocity distributions of $\mathrm{HF}$ and $\mathrm{p}-\mathrm{H}_{2} \mathrm{O}$ are also similar for $V_{\text {LSR }} \leqslant-85 \mathrm{~km} \mathrm{~s}^{-1}$, the optical depth profiles for $\mathrm{p}-\mathrm{H}_{2} \mathrm{O}$ are, however, significantly deeper than the HF optical depth profiles toward both sight lines.

To derive the $\mathrm{HF}$ and total $\mathrm{H}_{2} \mathrm{O}$ column densities, we first used a set of multiple Gaussian components to fit the $\mathrm{HF}$ and $\mathrm{p}-\mathrm{H}_{2} \mathrm{O}$ optical depth profiles over the LSR velocity range $[-200,+15] \mathrm{km} \mathrm{s}^{-1}$ for the components that showed optically thin or moderately thick HF absorption. To be conservative, we set a lower limit to the optical depth of $\tau=3$; our observations are not sensitive to larger optical depths (see Figure 1, right panels). A $\chi^{2}$ minimization showed that 16 components best fitted the HF optical depth profiles toward both sight lines for the velocity range selected here. Increasing the number of $\mathrm{HF}$ components in the fit did not significantly improve the $\chi^{2}$ values. Given the overall velocity distribution similarities between HF and $\mathrm{p}-\mathrm{H}_{2} \mathrm{O}$, we used the sight line cloud decomposition obtained from the HF fitting (FWHM and velocity integration range) as initial parameters to fit the $\mathrm{p}-\mathrm{H}_{2} \mathrm{O}$ optical depth distribution. Since the water absorption is significantly deeper than the HF absorption for $V_{\mathrm{LSR}} \leqslant-85 \mathrm{~km} \mathrm{~s}^{-1}$, we fixed the FWHM of the $\mathrm{p}-\mathrm{H}_{2} \mathrm{O}$ components to be those of $\mathrm{HF}$ and adopted the HF velocity integration ranges for the $\mathrm{p}-\mathrm{H}_{2} \mathrm{O}$ gas components (see Table 2) toward $\mathrm{G}-0.02-0.07$ and $\mathrm{G}-0.13-0.08$.

The best fit models to $\mathrm{HF}$ and $\mathrm{p}-\mathrm{H}_{2} \mathrm{O}$ are overlaid as green lines on the optical depth profiles for both sight lines in Figure 1 (right panels). We derived the $\mathrm{HF}$ and $\mathrm{p}-\mathrm{H}_{2} \mathrm{O}$ column densities for each velocity range listed in Table 2 following the method described in Neufeld et al. (2010). We assumed that the absorbing gas fully covers the continuum source and that each molecule is primarily in its ground state.

Table 2 lists, for each LSR velocity range considered here, $N(\mathrm{HF}), N\left(\mathrm{p}-\mathrm{H}_{2} \mathrm{O}\right), N(\mathrm{CH}), N\left(\mathrm{H}_{2} \mathrm{O}\right)_{\text {tot }} / N(\mathrm{HF})$ assuming the Galactic disk ortho/para ratio of 3 for $\mathrm{H}_{2} \mathrm{O}$ (Flagey et al. 2013), $N(\mathrm{HF}) / N(\mathrm{CH})$, a proxy for the $\mathrm{HF}$ gas-phase abundance relative to hydrogen in diffuse clouds, and $N\left(\mathrm{H}_{2} \mathrm{O}\right)_{\text {tot }} / N(\mathrm{CH})$. The errors are $2 \sigma$ unless otherwise noted. The last row reports the mean Galactic disk values for $N\left(\mathrm{H}_{2} \mathrm{O}\right)_{\text {tot }} / N(\mathrm{HF})$, for $N(\mathrm{HF}) / N(\mathrm{CH})$, and for $N\left(\mathrm{H}_{2} \mathrm{O}\right)_{\text {tot }} / N(\mathrm{CH})$.

\section{DISCUSSION}

Our analysis shows that for $-60 \leqslant V_{\mathrm{LSR}} \leqslant-45 \mathrm{~km} \mathrm{~s}^{-1}$ the $N\left(\mathrm{H}_{2} \mathrm{O}\right)_{\text {tot }} / N(\mathrm{HF})$ ratios are consistent with the mean Galactic disk diffuse ISM value of $1.5 \pm 0.4$ within $2 \sigma$. In contrast, the CMZ gas $\left(V_{\mathrm{LSR}} \leqslant-85 \mathrm{~km} \mathrm{~s}^{-1}\right)$ exhibits $N\left(\mathrm{H}_{2} \mathrm{O}\right)_{\text {tot }} / N(\mathrm{HF})$ ratios at least a factor of five larger than in the mean Galactic disk. 

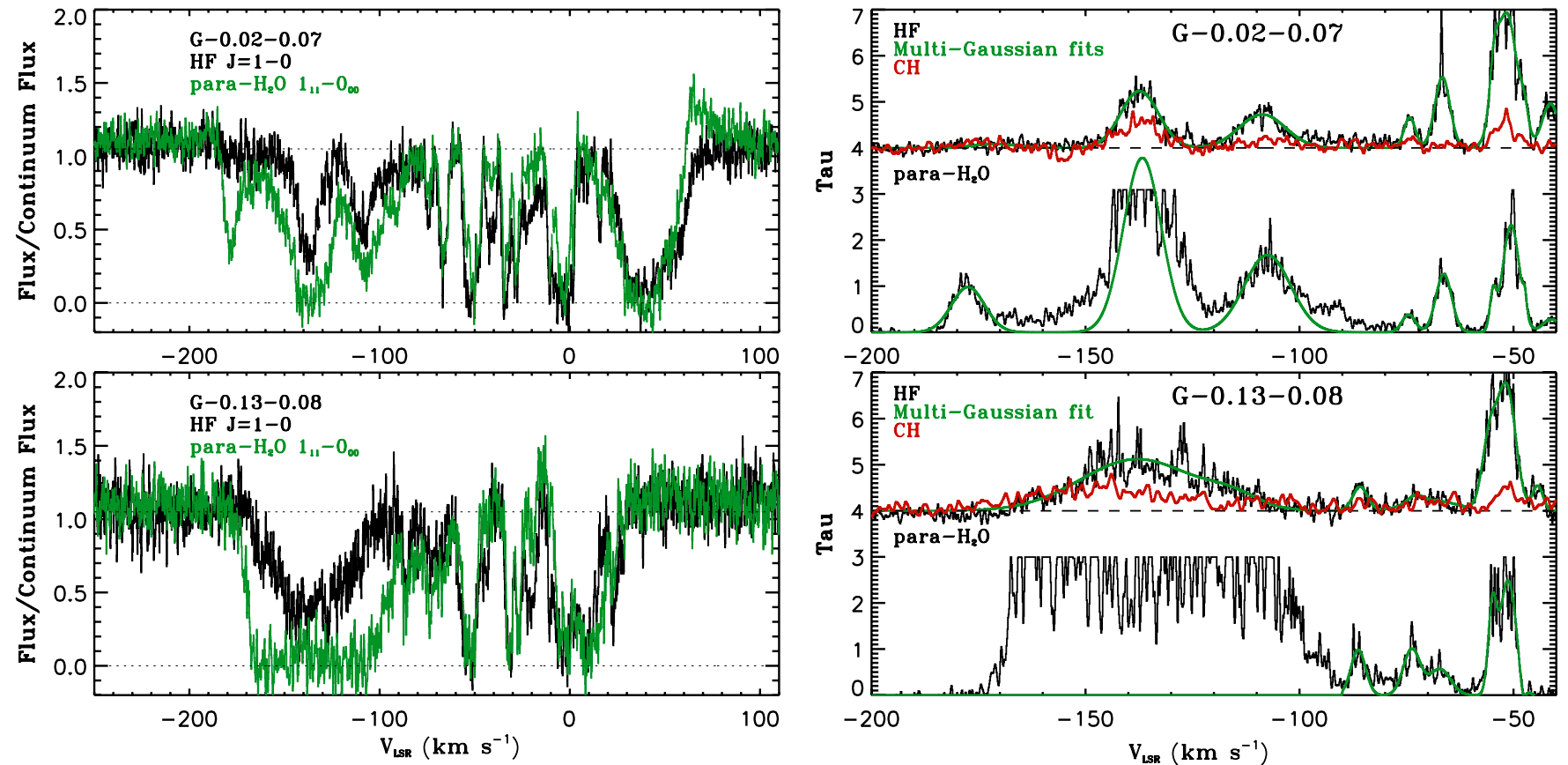

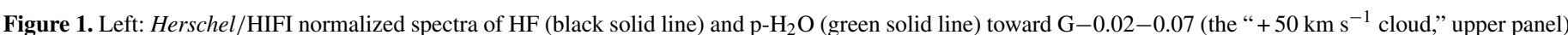

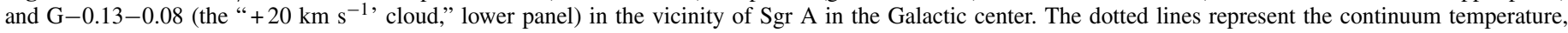

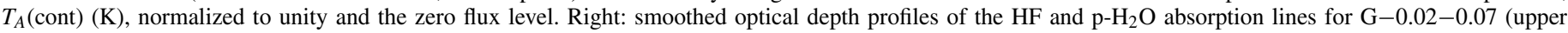

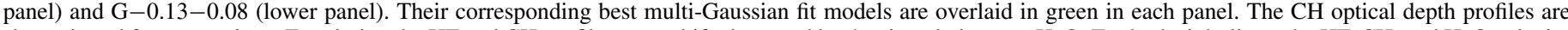

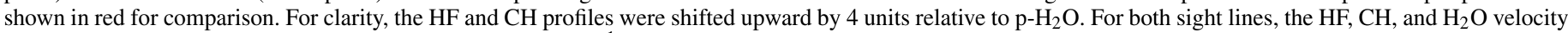

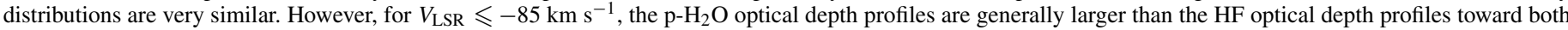
sight lines.

Table 2

Column Densities and Abundances

\begin{tabular}{|c|c|c|c|c|c|c|}
\hline $\begin{array}{l}V_{\mathrm{LSR}} \\
\left(\mathrm{km} \mathrm{s}^{-1}\right)\end{array}$ & $\begin{array}{c}N(\mathrm{HF}) \\
\left(10^{13} \mathrm{~cm}^{-2}\right)\end{array}$ & $\begin{array}{l}N\left(\mathrm{p}-\mathrm{H}_{2} \mathrm{O}\right) \\
\left(10^{13} \mathrm{~cm}^{-2}\right)\end{array}$ & $\begin{array}{c}N(\mathrm{CH}) \\
\left(10^{13} \mathrm{~cm}^{-2}\right)\end{array}$ & $\begin{array}{c}N\left(\mathrm{H}_{2} \mathrm{O}\right)_{\text {tot }^{\mathrm{a}}} \\
N(\mathrm{HF})\end{array}$ & $\begin{array}{l}N(\mathrm{HF}) \\
N(\mathrm{CH}) \\
\left(10^{-2}\right)\end{array}$ & $\begin{array}{c}N\left(\mathrm{H}_{2} \mathrm{O}\right)_{\text {tot }^{\mathrm{a}}} \\
N(\mathrm{CH})\end{array}$ \\
\hline \multicolumn{7}{|c|}{ Results for $\mathrm{G}-0.02-0.07$} \\
\hline$(-190,-170)$ & $\leqslant 0.5(3 \sigma)$ & $2.1 \pm 0.5$ & $5.6 \pm 1.4$ & $\geqslant 17(3 \sigma)$ & $\leqslant 15(3 \sigma)$ & $1.5 \pm 0.7$ \\
\hline$(-150,-130)$ & $3.5 \pm 0.7$ & $\geqslant 14$ & $24 \pm 6$ & $\geqslant 16$ & $14 \pm 5$ & $\geqslant 2.3$ \\
\hline$(-120,-90)$ & $2.7 \pm 0.6$ & $5.0 \pm 1.0$ & $8.9 \pm 2.6$ & $7.5 \pm 3.0$ & $30 \pm 10$ & $2.3 \pm 1.6$ \\
\hline$(-80,-70)$ & $0.58 \pm 0.17$ & $0.36 \pm 0.11$ & $2.8 \pm 0.7$ & $2.6 \pm 1.5$ & $21 \pm 9$ & $0.5 \pm 0.5$ \\
\hline$(-55,-45)$ & $5.2 \pm 1.6$ & $3.1 \pm 1.0$ & $15 \pm 5$ & $2.4 \pm 1.5$ & $33 \pm 16$ & $0.8 \pm 0.6$ \\
\hline \multicolumn{7}{|c|}{ Results for $\mathrm{G}-0.13-0.08$} \\
\hline$(-180,-107)$ & $9.9 \pm 1.7$ & $\geqslant 37$ & $79 \pm 16$ & $\geqslant 15$ & $13 \pm 5$ & $\geqslant 1.9$ \\
\hline$(-90,-80)$ & $0.54 \pm 0.15$ & $0.97 \pm 0.17$ & $3.8 \pm 0.9$ & $7.3 \pm 3.3$ & $14 \pm 7$ & $1.0 \pm 0.9$ \\
\hline$(-80,-60)$ & $0.78 \pm 0.12$ & $0.82 \pm 0.17$ & $5.7 \pm 1.3$ & $4.2 \pm 1.5$ & $14 \pm 7$ & $0.6 \pm 0.4$ \\
\hline$(-55,-40)$ & $5.4 \pm 1.1$ & $3.8 \pm 0.8$ & $14 \pm 4$ & $2.9 \pm 1.2$ & $38 \pm 17$ & $0.8 \pm 0.6$ \\
\hline \multicolumn{4}{|c|}{ Galactic Disk Mean } & $1.5 \pm 0.4^{\mathrm{b}}$ & $40 \pm 10^{c}$ & $0.6 \pm 0.3$ \\
\hline
\end{tabular}

Notes.

${ }^{\text {a }} N\left(\mathrm{H}_{2} \mathrm{O}\right)_{\text {tot }}$ is the total column density of water assuming the Galactic disk ortho/para ratio of 3 (see Flagey et al. 2013).

b Derived using absorptions detected toward W31C, W49N, and W51 (Sonnentrucker et al. 2010; Neufeld et al. 2010).

${ }^{c}$ Galactic mean value derived from velocity components detected toward W49N and W51 (Sonnentrucker et al. 2010; Gerin et al. 2010).

Herschel observations have established that HF can be used to trace molecular hydrogen in diffuse molecular clouds (e.g., Neufeld et al. 2010; Sonnentrucker et al. 2010) where its depletion onto dust grains is at m ost a factor of two with respect to solar abundances (Snow et al. 2007; Neufeld \& Wolfire, 2009; Guzmán et al. 2012) whereas HF depletes significantly in dense clouds (Neufeld et al. 2005; Phillips et al. 2010). Therefore, the higher-than-average $N\left(\mathrm{H}_{2} \mathrm{O}\right)_{\text {tot }} / N(\mathrm{HF})$ ratio at LSR velocities probing the $\mathrm{CMZ}$ could be indicative of the presence of dense molecular clouds where the HF depletion is larger compared to
$\mathrm{H}_{2} \mathrm{O}$. The presence of dense $\left(n_{\mathrm{H}}=10^{4} \mathrm{~cm}^{-3}\right)$, cold $(T=30 \mathrm{~K})$, and warm $(T \geqslant 400 \mathrm{~K})$ molecular clouds in the Galactic center is well established (e.g., Wilson et al. 1982; RodriguezFernandez et al. 2006) and has been extensively studied since then.

Alternatively, the HF abundance in the CMZ-associated gas could be consistent with the average Galactic disk diffuse gas abundance. The higher-than-average $N\left(\mathrm{H}_{2} \mathrm{O}\right)_{\text {tot }} / N(\mathrm{HF})$ would then indicate an enhanced $\mathrm{H}_{2} \mathrm{O}$ abundance by at least a factor of five in the CMZ. The $\mathrm{H}_{2} \mathrm{O}$ production could be enhanced 
compared to HF by endothermic reactions and reactions with energy barriers known to become efficient once $T \geqslant 400 \mathrm{~K}$ (e.g., Bergin et al. 1998; Neufeld et al. 2002). A warm diffuse molecular phase has been recently detected in the CMZ through observations of $\mathrm{H}_{3} \mathrm{O}^{+}$by Herschel (see D. Lis et al. 2013, in preparation) and observations of transitions out of the $(J, K)=$ $(1,1),(2,2)$, and $(3,3)$ states of $\mathrm{H}_{3}^{+}$; the population ratios of the latter states are predicted to be very sensitive to the gas temperature and density (see Oka \& Epp 2004) and were best reproduced toward the Galactic center with gas temperatures in the range $200-300 \mathrm{~K}$ and gas densities in the range $n_{\mathrm{H}}=$ 50-200 $\mathrm{cm}^{-3}$ (e.g., Goto et al. 2008, 2011; Oka et al. 2005).

To investigate whether HF is severely depleted or whether $\mathrm{H}_{2} \mathrm{O}$ is enhanced in diffuse clouds traced by $\mathrm{HF}$, we compared the HF column density distribution with that of another wellknown tracer of diffuse clouds, $\mathrm{CH}$. Previous observations have shown that in the diffuse cloud regime $N(\mathrm{CH}) \leqslant \sim$ few $\times 10^{14} \mathrm{~cm}^{-2}, \mathrm{CH}$ traces $\mathrm{H}_{2}$ linearly and $\mathrm{CH}$ does not exhibit significant depletion (e.g., Sheffer et al. 2008) contrary to the dense cloud regime (e.g., Mattila 1986). The groundstate doublet of the $\mathrm{CH}$ radical ( $v_{\text {rest }}=532 \mathrm{GHz}$ and $536 \mathrm{GHz}$ ) was also observed toward our sight lines with HIFI as part of the PRISMAS program. These data show a one-to-one correspondence between the $\mathrm{CH}$ and $\mathrm{HF}$ velocity distributions for the velocity range discussed here (see red optical depth profile in the right panels of Figure 1). We thus fitted the $\mathrm{CH}$ optical depth profiles using the strategy applied to $\mathrm{p}-\mathrm{H}_{2} \mathrm{O}$, after deconvolution of the $\mathrm{CH}$ hyperfine structure from the sight line velocity structures and derived the column densities following Gerin et al. (2010; see for details).

The $\mathrm{CH}$ column densities in the gas components associated with the $\mathrm{CMZ}$ fall in the regime where $\mathrm{CH}$ traces the presence of diffuse molecular clouds for both sight lines. $N(\mathrm{HF}) / N(\mathrm{CH})$ is thus expected to be equal to the measured Galactic disk mean value for the latter components. However, we find that $N(\mathrm{HF}) / N(\mathrm{CH})$ is lower than the mean Galactic disk value by a factor $\sim 2$ within our $2 \sigma$ uncertainties. This difference can either reflect a decrease in $N(\mathrm{HF})$ by factor of two resulting from mild HF depletion in these diffuse clouds, or it can reflect an increase in $N(\mathrm{CH})$ by the same factor. $\mathrm{CH}$ enhancements have been measured in the Galactic diffuse ISM (Federman 1982 and references therein) and are thought to result from formation involving $\mathrm{CH}^{+}$through warm gas-phase chemistry (e.g., Sheffer et al. 2008; Godard et al. 2009; Falgarone et al. 2010).

While our data do not allow us to lift the HF-CH degeneracy, our measurements certainly rule out the possibility that HF is severely depleted and traces the presence of dense clouds. Subsequently, we further conclude that the higher-than-average $N\left(\mathrm{H}_{2} \mathrm{O}\right)_{\text {tot }} / N(\mathrm{HF})$ in the CMZ-associated gas results from an enhanced water abundance by at least a factor of three (if $\mathrm{HF}$ is mildly depleted) or at least a factor of five (if $\mathrm{CH}$ is enhanced) with respect to the Galactic disk. Flagey et al. (2013) recently measured an $\mathrm{H}_{2} \mathrm{O}$ Galactic disk abundance of 5 $\times 10^{-8}$ based on a study of foreground gas components observed toward all seven PRISMAS sources. They also established that the $\mathrm{H}_{2} \mathrm{O}$ ortho/para ratio is equal to 3 within errors in the Galactic disk. For the cloud components absorbing in the range $[-55$ to -40$] \mathrm{km} \mathrm{s}^{-1}$ toward $\mathrm{G}-0.02-0.07$ and $\mathrm{G}-0.13-0.08$ we find $\mathrm{H}_{2} \mathrm{O}$ abundances similar to the Flagey et al. (2013) measurements, again indicating that these gas components mostly exhibit Galactic disk abundances whether they originate predominantly in the Galactic center region or in its foreground. For the velocity components consistent with CMZ-associated gas and showing enhanced water abundances, a lower limit to the water abundance is in the range $(15-33) \times 10^{-8}$.

The enhanced $\mathrm{H}_{2} \mathrm{O}$ (and potentially the $\mathrm{CH}$ ) abundances could result from production in warm (shock or X-ray) heated diffuse molecular gas (e.g., Martín-Pintado et al. 2000) where endothermic reactions and reactions with energy barriers then become efficient (see Bergin et al. 1998 for $\mathrm{OH}$ and $\mathrm{H}_{2} \mathrm{O}$; see Elitzur \& Watson 1978 and Falgarone et al. 2010 for $\mathrm{CH}^{+}$and $\mathrm{CH})$. It could also be a consequence of the known increases in elemental abundances measured toward the Galactic center compared to the Galactic disk. Various studies have concluded that the Galactic center $\mathrm{O} / \mathrm{H}$ ratio is typically higher by a factor of two compared to the disk gas (e.g., Cunha et al. 2007) and that the $\mathrm{C} / \mathrm{H}$ ratio is higher by at least a factor of three compared to the disk (e.g., Esteban et al. 2005 and references therein). While the increase in the $\mathrm{C}$ elemental abundance toward the Galactic center could certainly reproduce the $\mathrm{CH}$ enhancement potentially measured here, the increased $\mathrm{O}$ elemental abundance does not fully account for the water enhancement we derived.

Godard et al. (2012) recently reported large abundance enhancements compared to the Galactic disk for $\mathrm{CH}^{+}$and $\mathrm{SH}^{+}$ using HIFI observations over the entire negative velocity range probed by $\mathrm{p}-\mathrm{H}_{2} \mathrm{O}$ toward $\mathrm{G}-0.02-0.07 . \mathrm{CH}^{+}$and $\mathrm{SH}^{+}$are known to trace energetic processes (whether shocks or turbulent dissipation) in the diffuse ISM as their formation routes are highly endothermic, thereby prohibiting their efficient formation under diffuse-cloud physical conditions. Hence, the Godard et al. (2012) results lead us to suggest that energetic processes are the most likely origin of the abundance enhancement for both water and $\mathrm{CH}$ toward $\mathrm{G}-0.13-0.08$ and $\mathrm{G}-0.02-0.07$. One possible origin of the shocks is the strong velocity perturbations induced by the Galactic bar (see Ferrière 2012). Our observations therefore seem to support a picture in which both Galactic-disk-like diffuse molecular clouds traced by $\mathrm{HF}$ and warm $(T \geqslant 400 \mathrm{~K})$ heated diffuse molecular clouds revealed by the enhanced water and $\mathrm{CH}$ abundances are present within the inner $200 \mathrm{pc}$ region from the Galactic center, a picture previously proposed both via atomic and molecular absorption and emission line studies (e.g., Goto et al. 2008).

While $\mathrm{HF}$ and $\mathrm{p}-\mathrm{H}_{2} \mathrm{O}$ arise from well-separated clouds toward $\mathrm{G}-0.02-0.07$, their distribution is continuous toward $\mathrm{G}-0.13-0.08$ suggesting that the gas phases traced by both molecules occupy a large filling factor over a velocity range covering the CMZ. This picture is further supported by the detection of HF (Monje et al. 2011) and p- $\mathrm{H}_{2} \mathrm{O}$ (Lis et al. 2010) in diffuse clouds toward the Sgr B2 complex. Monje et al. found that the HF abundance was consistent with our Galactic mean value. They also noted that $N\left(\mathrm{H}_{2} \mathrm{O}\right)_{\text {tot }} / N(\mathrm{HF})=13.8$ toward Sgr B2 when using the Lis et al. (2010) p- $\mathrm{H}_{2} \mathrm{O}$ results in $\mathrm{CMZ}$-associated gas. Hence, the Sgr $\mathrm{B} 2 \mathrm{H}_{2} \mathrm{O}$ enhancement noted by Monje et al. is identical, within uncertainties, to the enhancement we report here for the Sgr A sight lines. The projected distance between the Sgr A and Sgr B2 cloud complexes is about $100 \mathrm{pc}$ assuming a distance to the Galactic center of $8.5 \mathrm{kpc}$. Hence, our results combined with the Monje et al. (2011) and Lis et al. (2010) results add weight to our suggestion that the diffuse medium traced by $\mathrm{HF}$ and $\mathrm{p}-\mathrm{H}_{2} \mathrm{O}$ is pervasive toward the Galactic center. It would be interesting to obtain follow-up observations of additional shock, X-ray heating, and cosmic ray tracers over the velocity range observed here to further study the origin of the warm diffuse molecular gas in the vicinity of the Galactic center. 
We wish to thank R. Güsten and M. Requena-Torres for extracting and sharing Herschel/HEXGAL data allowing us to determine that the OFF-beam position in our HIFI observations of $\mathrm{p}-\mathrm{H}_{2} \mathrm{O}$ and $\mathrm{HF}$ does not suffer from contamination due to the presence of extended emission/absorption around the Galactic center. We wish to thank our referee whose comments improved our work significantly. HIFI has been designed and built by a consortium of institutes and university departments from across Europe, Canada, and the United States under the leadership of SRON Netherlands Institute for Space Research, Groningen, The Netherlands, and with major contributions from Germany, France, and USA. Consortium members are: Canada: CSA, U. Waterloo; France: CESR, LAB, LERMA, IRAM; Germany: KOSMA, MPIfR, MPS; Ireland, NUI Maynooth; Italy: ASI, IFSI-INAF, Osservatorio Astrofisico di Arcetri-INAF; Netherlands: SRON, TUD; Poland: CAMK, CBK; Spain: Observatorio Astronómico Nacional (IGN), Centro de Astrobiología (CSIC/INTA); Sweden: Chalmers University of TechnologyMC2, RSS \& GARD, Onsala Space Observatory, Swedish National Space Board, Stockholm University-Stockholm Observatory; Switzerland: ETH Zurich, FHNW; USA: Caltech, JPL, NHSC. M.S. acknowledges support from grant N 203393334 from Polish MNiSW. Support for this work was provided to M. De Luca and M. Gerin by the Centre National de Recherche Spatiale (CNES) and by the SCHISM project (grant ANR-09BLAN-0231-01). J.R.G. is supported by a Ramón y Cajal research contract and thanks the Spanish MINECO for funding support through grants AYA2009-07304 and CSD2009-00038. Support for this work was provided by NASA through an award issued by JPL/Caltech.

Facility: Herschel

\section{REFERENCES}

Bergin, E. A., Neufeld, D. A., \& Melnick, G. J. 1998, ApJ, 499, 777

Cunha, K., Sellgren, K., Smith, V. V., et al. 2007, ApJ, 669, 1011

Dwarakanath, K. S., Goss, W. M., Zhao, J. H., \& Lang, C. C. 2004, JA\&A, 25, 129

Elitzur, M., \& Watson, W. D. 1978, ApJL, 222, 141

Esteban, C., García-Rojas, J., Peimbert, M., et al. 2005, ApJL, 618, 95
Falgarone, E., Godard, B., Cernicharo, J., et al. 2010, A\&A, 521, L15 Federman, S. R. 1982, ApJ, 257, 125

Ferrière, K. 2012, A\&A, 540, A50

Flagey, N., Goldsmith, P. F., Lis, D. C., et al. 2013, ApJ, 762, 11

Gerin, M., de Luca, M., Goicoechea, J. R., et al. 2010, A\&A, 521, L16

Genzel, R., Stacey, G. J., Harris, A. I., et al. 1990, ApJ, 356, 160

Godard, B., Falgarone, E., Gerin, M., et al. 2012, A\&A, 540, A87

Godard, B., Falgarone, E., \& Pineau Des Forêts, G. 2009, A\&A, 495, 847

Goto, M., Usuda, T., Geballe, T. R., et al. 2011, PASJ, 63, L13

Goto, M., Usuda, T., Nagata, T., et al. 2008, ApJ, 688, 306

Guesten, R., Walmsley, C. M., \& Pauls, T. 1981, A\&A, 103, 197

Guzmán, V., Pety, J., Gratier, P., et al. 2012, A\&A, 543, L1

Lang, C. C., Goss, W. M., Cyganowski, C., \& Clubb, K. I. 2010, ApJS, 191,275

Lis, D. C., Phillips, T. G., Goldsmith, P. F., et al. 2010, A\&A, 521, L26

Liszt, H. S., Burton, W. B., \& van der Hulst, J. M. 1985, A\&A, 142, 237

Liszt, H. S., van der Hulst, J. M., Burton, W. B., \& Ondrechen, M. P. 1983, A\&A, 126, 341

Martín-Pintado, J., de Vicente, P., Rodríguez-Fernández, N. J., Fuente, A., \& Planesas, P. 2000, A\&A, 356, L5

Mattila, K. 1986, A\&A, 160, 157

Monje, R. R., Emprechtinger, M., Phillips, T. G., et al. 2011, ApJL, 734, 23

Neufeld, D. A., Kaufman, M. J., Goldsmith, P. F., Hollenbach, D. J., \& Plume, R. 2002, ApJ, 580, 278

Neufeld, D. A., Sonnentrucker, P., Phillips, T. G., et al. 2010, A\&A, 518, L108

Neufeld, D. A., \& Wolfire, M. G. 2009, ApJ, 706, 1594

Neufeld, D. A., Wolfire, M. G., \& Schilke, P. 2005, ApJ, 628, 260

Oka, T., \& Epp, E. 2004, ApJ, 613, 349

Oka, T., Geballe, T. R., Goto, M., Usuda, T., \& McCall, B. J. 2005, ApJ, 632,882

Oka, T., Nagai, M., Kamegai, K., \& Tanaka, K. 2011, ApJ, 732, 120

Ott, S. 2010, in ASP Conf. Ser. 434, Astronomical Data Analysis Software and Systems XIX, ed. Y. Mizumoto, K.-I. Morita, \& M. Ohishi (San Francisco, CA: ASP), 139

Phillips, T. G., Bergin, E. A., Lis, D. C., et al. 2010, A\&A, 518, L109

Pilbratt, G. L., Riedinger, J. R., Passvogel, T., et al. 2010, A\&A, 518, L1

Rodriguez-Fernandez, N. J., Combes, F., Martin-Pintado, J., Wilson, T. L., \& Apponi, A. 2006, A\&A, 455, 963

Sawada, T., Hasegawa, T., Handa, T., \& Cohen, R. J. 2004, MNRAS, 349, 1167

Sheffer, Y., Rogers, M., Federman, S. R., et al. 2008, ApJ, 687, 1075

Snow, T. P., Destree, J. D., \& Jensen, A. G. 2007, ApJ, 655, 285

Sonnentrucker, P., Neufeld, D. A., Phillips, T. G., et al. 2010, A\&A, 521, L12

Wilson, T. L., Ruf, K., Walmsley, C. M., et al. 1982, A\&A, 115, 185

Wright, M. C. H., Coil, A. L., McGary, R. S., Ho, P. T. P., \& Harris, A. I. 2001, ApJ, 551, 254 